Rev. Elev. Méd. vét. Pays trop., 1972, 25 (3) : 445-454

\title{
Etude de la mortalité bovine au Centre de Recherches Zootechniques de Dara (Sénégal)
}

\author{
par J. P. DENIS $\left(^{*}\right)$ et J. VALENZA $\left(^{*}\right)$
}

\begin{abstract}
RESUME
Le taux moyen annuel de mortalité bovine au C.R.Z. de Dara est faible: 6,21 p. $100 ; 1,9$ p. 100 de la population meurt avant d'avoir atteint 6 mois; 3,25 p. 100 avant 1 an et 5 p. 100 avant deux ans. 26 p. 100 des morts ont moins de 50 jours, 29 p. 100 entre 6 et 12 mois, 80 p. 100 moins de 2 ans.

Le poids et l'époque de naissance et le poids au sevrage ont une influence certaine sur cette mortalité, de même que la saison: c'est entre avril et août et surtout en juillet que la mortalité est la plus élevée; mais cette période correspond à celle où il y le plus de naissances. D'autre part, les mâles sont moins sensibles.

Les causes principales de mortalité sont constituées par les accidents septicémiques survenant chez les jeunes veaux, et les accidents survenant après le sevrage.
\end{abstract}

\section{INTRODUCTION}

Le bilan économique d'un élevage bovin tient compte pour une large part de la mortalité potentielle dans le troupeau.

Deux périodes sont critiques : celle immédiatement après la naissance, puis celle après le sevrage. Cette dernière est particulièrement importante compte tenu des conditions alimentaires précaires de la zone sylvo-pastorale du Sénégal.

Annuellement, dans les troupeaux de cette zone, la mortalité chez les jeunes peut atteindre 30 à 40 p. 100 des naissances, et même 50 p. 100 durant les années où l'hivernage a été particulièrement pauvre. Par contre, chez les adultes, la mortalité a été fortement dimi-

(*) Centre de Recherches zootechniques de DaraDjoloff, Laboratoire national de l'Elevage et de Recherches vétérinaires, B.P. no 2057, Dakar-Hann, Sénégal. nuée par l'application des moyens de prophylaxie systématique.

Au C.R.Z. de Dara, les vaccinations sont régulièrement pratiquées, et il apparaît aussi que la mortalité est surtout importante chez les animaux jeunes.

Il a semblé, par conséquent, intéressant d'étudier l'évolution de cette mortalité, les facteurs de variation entrant en jeu, la répartition selon l'âge des animaux dans les conditions de vie du C.R.Z. de Dara déjà définies (1).

L'étude dans son ensemble couvre une période de 16 années. Elle sera divisée en trois parties principales. Dans une première partie, seront étudiées les causes de mortalité sur le plan pathologique. L'analyse ne portera cependant pas sur l'ensemble de la période annoncée car, pour un certain nombre d'années, les informations sont insuffisantes. Mais néanmoins, les principales causes responsables de la mortalité sont nettement perceptibles. 
La seconde partie sera consacrée à l'analyse numérique de la mortalité, de sa répartition, de l'action de différents facteurs sur son évolution.

Dans la troisième partie enfín, la mortalité chez les jeunes animaux sera particulièrement étudiée.

\section{LES CAUSES DE MORTALITE}

Cette étude mérite quelques précisions préliminaires.

Les buts des actions zootechniques entreprises au C.R.Z. de Dara ont imposé la nécessité de ne pas s'éloigner trop des conditions d'entretien des animaux à l'extérieur pour que les géniteurs cédés aux éleveurs à partir du Centre puissent s'y adapter. Par conséquent, le mode d'entretien adopté consiste en une gestion tenant compte davantage de ces conditions (en particulier climatiques) que de méthodes faisant intervenir, entre autres, la supplémentation alimentaire systématique.

Aussi, un pourcentage important des causes de mortalité est la conséquence directe au cours des années examinées des considérations précédentes.

Les différentes causes de mortalité observées apparaissent au tableau $\mathrm{n}^{\circ}$ I. Elles sont exprimées en pourcentage de la mortalité globale par ordre de valeur décroissante:

TABLEAU $\mathrm{N}^{\circ} \mathrm{I}$

\begin{tabular}{|l|c|}
\hline & Pourcentage \\
\cline { 2 - 2 } Septicémie des jeunes & 22,8 \\
Accidents au sevrage & 18,8 \\
Charbon & 10,7 \\
Peste & 10,7 \\
Anaplasmose & 7,3 \\
Accidents & 5,9 \\
Mortinatalité & 2,9 \\
Agalactie maternelle & 2,9 \\
Envenimation ophidienne & 2,9 \\
Botuligme & 2,5 \\
Indigestion aiguë & 2,2 \\
Rage & 1,8 \\
Enterotoxêmie & 1,4 \\
Cachexie sénile & 1,1 \\
Actinomycose & 0,7 \\
Pneumonie gangréneuse & 0,7 \\
Bronchopneumonie par corps étranger & 0,7 \\
Tuberculose (sacrifice) & 0,3 \\
Météorisation & 0,3 \\
Congestion pulmonaire & 0,3 \\
Non diagnostiqués & 2,9 \\
& \\
\hline
\end{tabular}

De ce tableau, il ressort que l'essentiel de la mortalité est causé par les accidents atteignant les jeunes animaux, à savoir :

— septicémie . . . . . . . 22,8

一 agalactie . . . . . . . .

- mortinatalité . . . . . 2,9

— accidents au sevrage . . . . . 18,8

soit environ 47 p. 100 de la mortalité totale.

Une mention particulière doit être faite pour les cas de mortalité dus au charbon symptomatique, à la peste et au botulisme. Ces mortalités constituent des accidents qui ne se produisent plus à partir du moment où des mesures de prophylaxie rigoureuses sont appliquées et, par conséquent, ont une faible importance. Dans le cas de l'anaplasmose, il s'agit d'une invasion brutale qui a été circonscrite rapidement une fois le diagnostic effectué et le traitement approprié instauré.

Si l'on fait abstraction des pourcentages relatifs à ces dernières maladies, celui des affections touchant les jeunes animaux devient environ 69 p. 100 , soit une valeur proche de celle indiquée en fonction de la classe d'âge (80 p. 100 des morts surviennent entre la naissance et 24 mois, chapitre III).

Le facteur "accidents au sevrage " est responsable de la mort de nombreux animaux durant la fin de la saison sèche et le début de la saison des pluies. Faute d'une alimentation suffisante en quantité et qualité, les animaux maigrissent, leur résistance diminue et ils meurent rapidement sans signes prémonitoires. Les infections bactériennes de sortie sont très rares; on peut constater, par contre, un parasitisme interne élevé.

Les moyens de lutte, actuellement employés, sont :

1. Sur le plan pathologique, la vaccination systématique des jeunes animaux contre la septicémie à l'aide d'un stock-vaccins (coliPasteurella). Mais cette mesure semble encore insuffisante et la fabrication d'un auto-vaccin d'étable est envisagée;

2. Sur le plan nutritionnel, les veaux reçoivent un concentré titrant 0,80 U.F. et $90 \mathrm{~g}$ de MAD par $\mathrm{kg}$ à raison de $500 \mathrm{~g}$ à $1 \mathrm{~kg}$ par tête et par jour en fonction de l'avancement de la saison sèche, et les vaches sélectionnées, $1 \mathrm{~kg}$ par jour de ce même concentré dans un 
TABLEAU $N^{\circ}$ II

Evolution des effectıfs de 1954 à 1969 - Causes de variations

\begin{tabular}{|c|c|c|c|c|c|c|c|c|c|c|c|c|c|c|c|c|c|}
\hline & & 54 & 55 & 56 & 57 & 58 & 59 & 60 & 61 & 62 & 63 & 64 & 65 & 66 & 67 & 68 & 69 \\
\hline \multirow{3}{*}{ Males } & Naissunces annuelles & 20 & 41 & 69 & 88 & 113 & 130 & 111 & 153 & 140 & 111 & 126 & 97 & 80 & 79 & 57 & 69 \\
\hline & Nalssances cumulées & 20 & 61 & 130 & 218 & 331 & 461 & 572 & 725 & 865 & 976 & 1102 & 1199 & 1279 & 1358 & 1415 & 1484 \\
\hline & ELEectje 31.12 & 27 & 67 & 129 & 206 & 249 & 263 & 304 & 197 & 282 & 224 & 283 & 197 & 150 & 148 & 172 & 203 \\
\hline \multirow{3}{*}{ Femeiles } & Naissances dnnuelles & 23 & 42 & 71 & 88 & 94 & 139 & 131 & 134 & 128 & 96 & 124 & 101 & 90 & 93 & 71 & 61 \\
\hline & Naissances cumulēes & 23 & 65 & 136 & 224 & 318 & 457 & 588 & 722 & 850 & 946 & 1070 & 1171 & 1261 & 1354 & 1425 & 1486 \\
\hline & Effectif 31.12 & 249 & 282 & 344 & 418 & 492 & 587 & 646 & 672 & 711 & 648 & 687 & 462 & 480 & 443 & 442 & 421 \\
\hline \multirow{3}{*}{ Total } & Naissances annuelles & 43 & 83 & 140 & 176 & 207 & 269 & 242 & 287 & 268 & 207 & 250 & 198 & 170 & 172 & 128 & 130 \\
\hline & Naissances cumulêes & 43 & 126 & 266 & 442 & 649 & 918 & 1160 & 1447 & 1715 & 1922 & 2172 & 2370 & 2540 & 2712 & 2840 & 2970 \\
\hline & Effectif 31.12 & 276 & 349 & 473 & 624 & 741 & 850 & 950 & 869 & 993 & 872 & 970 & 659 & 630 & 591 & 614 & 624 \\
\hline \multirow{3}{*}{$\begin{array}{l}\text { Variations } \\
\text { des elfec } \\
\text { tifs en } \\
(+)\end{array}$} & Naussances annuelles & 43 & 83 & 140 & 176 & 207 & 269 & 242 & 287 & 268 & 207 & 250 & 198 & 170 & 172 & 128 & 130 \\
\hline & Naissances cumulées & 43 & 126 & 266 & 442 & 649 & 918 & 1160 & 1447 & 1715 & 1922 & 2172 & 2370 & 2540 & 2712 & 2840 & 2970 \\
\hline & Achats & 101 & 1 & - & - & - & - & - & - & - & - & - & - & - & - & - & - \\
\hline \multirow{4}{*}{$\begin{array}{l}\begin{array}{l}\text { Variations } \\
\text { des }\end{array} \\
\text { effectirs } \\
(-)\end{array}$} & RéEormês & - & - & - & - & 28 & 110 & 66 & 247 & 98 & 199 & 98 & 461 & 117 & 118 & 24 & 101 \\
\hline & Morts & 6 & 11 & 16 & 25 & 42 & 50 & 75 & 106 & 46 & 123 & 47 & 29 & 76 & 61 & 50 & 14 \\
\hline & Cessions & - & - & - & - & 20 & - & 1 & 15 & - & 6 & 7 & 19 & 6 & 32 & 31 & 5 \\
\hline & Total & 6 & 10 & 16 & 25 & 90 & 160 & 142 & 368 & 144 & 328 & 152 & 509 & 199 & 211 & 105 & 120 \\
\hline
\end{tabular}


double but: participation aux besoins d'entretien et aux besoins de fin de gestation.

L'effet de ces quelques mesures, particulièrement chez les animaux après le sevrage, a permis de diminuer le taux de mortalité qui est devenu 1,88 p. 100 en 1969 et 2,2 p. 100 en 1970.

\section{EVOLUTION GENERALE DU TROUPEAU DE 1954 A 1969}

\section{Effectifs :}

Leur évolution, pour une période s'étendant sur 15 années consécutives (1954-1969), est concrétisée dans le tableau $\mathrm{n}^{\circ} \mathrm{II}$ (p. 447).

L'étude de cette évolution montre:

- que pour 2.470 naissances cumulées, la proportion des mâles est de 49,96 p. 100 pour 50,04 de femelles;

- que la courbe des naissances évolue de façon parallèle à celle des effectifs totaux des femelles, ce qui est l'indice d'une continuité certaine dans les taux annuels do vêlage;

- que pour diverses années - de 1961 à 1964 notamment, il existe un certain parallélisme entre les taux de réforme et de mortalité, ce qui indique - compte tenu des conditions climatiques donc alimentaires défavorables provoquant un accroissement périodique de la mortalité - une baisse générale de l'état des animaux avec comme possible incidence des réformes peut-être prématurées.

Il est par conséquent nécessaire, sauf dans des cas particuliers, de maintenir un âge fixe pour les jugements de sélection sur la croissance; cet âge fixe donne aux animaux le maximum de chances de faire preuve de leurs capacités propres en éliminant autant que possible l'influence des variations climatiques annuelles dont l'importance est extrême.

Répartition annuelle de la mortalité.

Dans l'ensemble, l'évolution de la mortalité suit celle des effectifs globaux. Cependant, certaines années, comme 1960,1961, 1963 et 1966 apparaissent comme très néfastes du fait notamment de saisons des pluies particulièrement courtes aux précipitations peu abondantes et de la pathologie.

Le taux moyen annuel de mortalité est de 6,23 p. 100 sur 15 années, de 1955 à 1969 (tableau $\mathrm{n}^{\circ}$ III).

TABLEAU N ${ }^{\circ}$ III

Evolution du taux annuel de mortalité

\begin{tabular}{|l|l|l|l|l|l|l|l|l|l|l|l|l|l|l|}
\hline 1955 & 1956 & 1957 & 1958 & 1959 & 1960 & 1961 & 1962 & 1963 & 1964 & 1965 & 1966 & 1967 & 1968 & 1969 \\
\hline 3,06 & 3,27 & 3,85 & 5,05 & 4,95 & 6,86 & 8,56 & 4,04 & 10,25 & 4,18 & 2,48 & 9,16 & 7,60 & 6,95 & 1,88 \\
\hline
\end{tabular}

Répartition mensuelle de la mortalité.

Sur dix années, de 1960 à 1969, la répartition mensuelle de la mortalité apparaît sur le tableau $\mathbf{n}^{\circ}$ IV.

La mortalité maximale a lieu au cours du mois de juillet. Cette observation recoupe celles concernant la diminution très importante de poids des animaux adultes en juillet (1) qui est concomitant d'un affaiblissement général des défenses organiques.

307 morts sur 627 , soit 48,9 p. 100 , ont lieu entre avril et juillet, durant la deuxième partie de la saison sèche. La période favorable se situe entre août et novembre.

\begin{tabular}{|c|c|c|c|c|c|c|c|c|c|c|c|c|c|}
\hline \multirow{3}{*}{ Nombre } & 1 & 2 & 3 & 4 & 5 & 6 & 7 & 8 & 9 & 10 & 11 & 12 & Tota1 \\
\cline { 2 - 21 } & 47 & 38 & 45 & 63 & 73 & 79 & 92 & 45 & 35 & 29 & 32 & 49 & 627 \\
\hline Pourcentage & 7,4 & 6,0 & 7,1 & 10,0 & 11,6 & 12,5 & 14,6 & 7,1 & 5,5 & 4,6 & 5,1 & 7,8 & 100 \\
\hline
\end{tabular}


Répartition de la mortalité en fonction de la classe d'âge

- Par rapport au nombre d'animaux morts
Cette répartition apparaît au tableau $\mathbf{n}^{\circ} \mathrm{V}$.

Sur la totalité des animaux morts en 10 ans, 52,1 p. 100 meurent avant un an et 80 p. 100 avant deux ans.

TABLEAU $\mathrm{N}^{\circ} \mathrm{V}$

\begin{tabular}{|c|c|c|c|c|c|c|c|}
\hline & \multirow{2}{*}{$A g e s$} & \multirow{2}{*}{ Nombre } & \multirow{2}{*}{ Pourcentage } & \multicolumn{2}{|c|}{$0 \mathrm{~d} 24$ mols } & \multicolumn{2}{|c|}{0 à 12 mois } \\
\hline & & & & Nombre & Pourcentage & Nombre & Pourcentage \\
\hline 1 & 0 à 6 mois & 191 & 30,4 & \multirow{3}{*}{503} & \multirow{3}{*}{80,2} & \multirow[t]{2}{*}{327} & \multirow[t]{2}{*}{52,1} \\
\hline 2 & 6 à 12 mois & 136 & 21,6 & & & & \\
\hline 3 & 12 a 24 mois & 176 & 28,0 & & & \multirow{4}{*}{300} & \multirow{4}{*}{47,8} \\
\hline 4 & 2 à 3 ans & 30 & 4,7 & \multirow{3}{*}{124} & \multirow{3}{*}{19,7} & & \\
\hline 5 & 3 à 4 ans & 17 & 2,7 & & & & \\
\hline 6 & $>4$ ans & 77 & 12,2 & & & & \\
\hline \multicolumn{2}{|c|}{$T \circ t a l$} & 627 & 100 & \multicolumn{2}{|c|}{2 ans $a ̀>$} & \multicolumn{2}{|c|}{1 an à > } \\
\hline
\end{tabular}

- Par rapport à la totalité du troupeau pour les années 60 à 69

A la lecture du tableau $\mathbf{n}^{\mathbf{0}}$ VI, il apparait que 1,90 p. 100 de l'ensemble de la population meurt avant d'avoir atteint ses premiers 6 mois, 3,25 p. 100 avant d'avoir atteint la première année, et 5 p. 100 avant la deuxième année.

\section{- En fonction du trimestre de mortalité}

Il s'agit de mettre en évidence les classes d'âge plus particulièrement sensibles à la saison sèche.
TABLEAU $\mathrm{N}^{\circ} \mathrm{VI}$

\begin{tabular}{|c|c|c|}
\hline Age s & Nombre & Pourcentage \\
\hline $0-6$ & 191 & 1,90 \\
\hline $6-12$ & 136 & 1,35 \\
\hline $12-24$ & 176 & 1,75 \\
\hline $24-36$ & 30 & 0,29 \\
\hline $3-4$ & 17 & 0,16 \\
\hline 4 et plus & 77 & 0,76 \\
\hline T o t a 1 & 627 & 6,21 \\
\hline
\end{tabular}

TABLEAU $N^{\circ}$ VII

\begin{tabular}{|c|c|c|c|c|c|c|c|c|c|}
\hline \multirow{2}{*}{$A \mathrm{~g} e \mathrm{~s}$} & \multicolumn{2}{|c|}{$1^{\text {er }}$ trimestre } & \multicolumn{2}{|c|}{$2 e$ trimestre } & \multicolumn{2}{|c|}{ 3e trimestre } & \multicolumn{2}{|c|}{$4 \mathrm{e}$ trimestre } & \multirow{2}{*}{ Total } \\
\hline & Nombre & Pourcentage & Nombre & Pourcentage & Nombre & Pourcentage & Nombre & Pourcentage & \\
\hline $0-6$ & 39 & 20,4 & 61 & 31.9 & 54 & $2 B, 3$ & 37 & 19,4 & 191 \\
\hline $6-12$ & 39 & 28,6 & 56 & 41.1 & 18 & 13,2 & 23 & 16,9 & 136 \\
\hline $12-24$ & 21 & 11.9 & 61 & 34,6 & 60 & 34,5 & 34 & 19,3 & 176 \\
\hline $24-36$ & 8 & 26,6 & 4 & 13,3 & 13 & 43,3 & 5 & 16,6 & 30 \\
\hline $3-4$ & 3 & 17,6 & 5 & 19,4 & 6 & 35.2 & 3 & 17,6 & 17 \\
\hline 4 et plus & 18 & 23,3 & 31 & 40,2 & 20 & 25,9 & 8 & 10,3 & 77 \\
\hline & & & & & & & & & 627 \\
\hline
\end{tabular}

A la lecture du tableau $n^{\circ}$ VII, il apparaît que dans l'ensemble, au cours des $2^{\text {e }}$ et $3^{\text {e }}$ trimestres, quelle que soit la tranche d'âge considérée, la mortalité est maximale. Donc, c'est essentiellement pendant la fin de la saison sèche et le début de la saison des pluies qu'on observe le maximum de morts. Il faut cependant signaler que cette mortalité est minimale 
pendant le $3^{\mathrm{e}}$ trimestre pour la période du post-sevrage.
Répartition de la mortalité en fonction du sexe

TABLEAU N ${ }^{\circ}$ VIII

\begin{tabular}{|l|c|c|c|c|c|c|c|c|c|c|c|c|c|}
\hline & 1 & 2 & 3 & 4 & 5 & 6 & 7 & 8 & 9 & 10 & 11 & 12 & Total \\
\hline Mâles & 21 & 15 & 21 & 22 & 33 & 34 & 48 & 18 & 10 & 12 & 12 & 27 & 273 \\
\hline Femelles & 26 & 23 & 24 & 41 & 40 & 45 & 44 & 27 & 25 & 17 & 20 & 22 & 354 \\
\hline
\end{tabular}

Durant les dix dernières années, la mortalité femelle est de 56,4 p. 100 contre 43,5 p. 100 de mortalité mâle, et la différence entre les deux taux est significative. Donc dans l'ensemble, les femelles sont plus sensibles que les mâles aux diverses agressions.
En ce qui concerne l'évolution des pourcentages respectifs mâles et femelles au cours de l'année, les résultats sont donnés dans le tableau $\mathrm{n}^{\circ}$ IX suivant:

TABLEAU $\mathrm{N}^{\circ}$ IX

\begin{tabular}{|l|c|c|c|c|c|c|c|c|c|c|c|c|c|}
\hline & 1 & 2 & 3 & 4 & 5 & 6 & 7 & 8 & 9 & 10 & 11 & 12 & \\
\hline Mâles & 3,3 & 2,3 & 3,3 & 3,5 & 5,2 & 5,4 & 7,6 & 2,8 & 1,5 & 1,9 & 1,9 & 4,3 & 627 \\
\hline Feñlles & 4,1 & 3,6 & 3,8 & 6,5 & 6,3 & 7,1 & 7,0 & 4,3 & 3,9 & 2,7 & 3,1 & 3,5 & 627 \\
\hline
\end{tabular}

Il apparaît que la répartition dans l'année des mortalités femelles est régulière avec une augmentation apparaissant dès le mois d'avril et durant jusqu'en juillet.

Quant aux mâles, il y a une augmentation régulière du mois de février à juin, un pic au mois de juillet, puis une descente très brusque au mois d'août.

Ceci semblerait indiquer qu'une brusque aggravation des conditions écologiques éprouve plus durement les mâles que les femelles.

\section{ETUDE PARTICULIERE DE LA MORTALITE DE LA NAISSANCE A 24 MOIS}

80 p. 100 des mortalités ont lieu entre la naissance et deux ans; 5 p. 100 de la population meurt avant d'avoir atteint sa deuxième année, c'est dire l'importance de cette période et justifier son étude spéciale.

La répartition de cette mortalité est tout d'abord étudiée semaine par semaine pour mettre en évidence les points critiques.
Puis l'étude d'un certain nombre de facteurs est abordée :

- pour la période post-natale :

— poids à la naissance,

- sexe,

- époque de naissance.

- pour la période post-sevrage :

- poids au sevrage,

- époque.

Répartition de la mortalité de 0 à 24 mois.

Les données recueillies permettent de faire les remarques suivantes:

a) la mortalité est maximale entre la naissance et 50 jours : 26 p. 100 du total dont 12,3 p. 100 durant les 45 premiers jours;

b) elle diminue progressivement jusqu'au sevrage effectué à 6 mois;

c) de 6 à 12 mois (29 p. 100) apparaît un taux élevé dont le maximum se situe à la $46^{\mathrm{e}}$ semaine, soit 10 mois et demi;

d) de 12 à 18 mois, la mortalité diminaue progressivement mais reste relativement élevée (24,4 p. 100 du total des morts). Ceci peut être imputé au mode d'élevage. En effet, à 
12 mois, les animaux mâles et femelles sont séparés et inclus respectivement dans les troupeaux de taureaux - taurillons et de génisses. Ils y trouvent des animaux beaucoup plus âgés, donc mieux armés sur le plan de la concurrence vitale. Il s'agit d'une sorte de " second sevrage» auquel il est difficile d'échapper compte tenu des possibilités actuelles d'utilisation des pâturages du Centre.

Elles montrent également que la mortalité durant les deux premiers jours représente 3,1 p. 100 des morts de la naissance à 24 mois et 23,8 p. 100 des animaux morts durant les premiers 15 jours qui représentent 13,3 p. 100 du total.

Les pourcentages de mortalité en fonction du mois de naissance figurent au tableau $n^{0} X$.

TABLEAU $\mathrm{N}^{\circ} \mathrm{X}$

\begin{tabular}{|c|c|c|}
\hline $\begin{array}{c}\text { Mois de } \\
\text { naissance }\end{array}$ & $\begin{array}{c}\text { Nombre } \\
\text { d'animaux morts }\end{array}$ & Pourcentag \\
\hline 1 & 28 & 5,7 \\
2 & 40 & 8,2 \\
3 & 40 & 8,2 \\
4 & 33 & 6,7 \\
5 & 64 & 13,1 \\
6 & 80 & 16,4 \\
7 & 76 & 15,6 \\
8 & 43 & 8,8 \\
9 & 27 & 5,5 \\
10 & 14 & 2,8 \\
11 & 13 & 2,6 \\
12 & 28 & 5,7 \\
\hline T o a & 486 & 100 \\
\hline
\end{tabular}

Si l'on compare cette répartition par rapport à celle des naissances, on observe qu'à la plus forte mortalité, qui intervient entre mai et juillet correspond le maximum des naissances.

Ces données produites en courbes donnent des traits qui coïncident à peu près parfaitement, ce qui permet d'affirmer que le mois de naissance n'a que peu d'influence sur la mortalité des jeunes avant l'âge de deux ans.

\section{Facteurs influençant cette mortalité}

Différents facteurs peuvent agir sur le taux de mortalité avant deux ans durant deux périodes de la vie des animaux.

Pendant la première, de la naissance au sevrage (6-7 mois), poids, mois de naissance et sexe agissent; au cours de la deuxième (après sevrage), ce sont le poids de sevrage et la période de naissance.

\section{Mortalité entre 0 et 6 mois}

\section{Poids à la naissance}

Il semble agir sur la mortalité avant sevrage. En effet, le poids moyen à la naissance des animaux morts entre 1960 et 1969 avant le sevrage est de $20,480 \mathrm{~kg}$ (115 animaux) contre $21,590 \mathrm{~kg}$ pour l'ensemble des produits (2.052 animaux) nés durant la même période. Il existe donc une différence de $1,1 \mathrm{~kg}$. Ceci conduit à penser que, chez les animaux les moins lourds à la naissance, la mortalité est supérieure pendant la période de pré-sevrage. Toutefois, ces résultats ne peuvent être considérés d'une façon absolue car de nombreux animaux, mourant très rapidement dès leur naissance, ne sont pas pesés, ce qui introduit un facteur de variation non contrôlé dans l'observation.

\section{Période de naissance}

Si l'on compare les taux d'animaux morts entre 0 et 6 mois par rapport aux naissances pendant chaque trimestre, il apparaît que le taux est maximal durant le quatrième. Sachant que cette mortalité est la plus forte durant les deux premiers mois de la vie de l'animal, l'influence du trimestre peut être considérée comme certaine et directe.

Ce taux supérieur du quatrième trimestre, peut être dû, malgré des conditions alimentaires des mères plus favorables, à un développement accru de parasitisme interne des jeunes durant la période qui suit la fin de la saison des pluies (hypothèse à vérifier), tableau $n^{\circ}$ XI.

TABLEAU $\mathrm{N}^{\bullet} \mathrm{XI}$

\begin{tabular}{|c|c|c|c|}
\hline Trimestre & $\begin{array}{c}\text { Nombre } \\
\text { de morts }\end{array}$ & $\begin{array}{c}\text { Nombre de } \\
\text { naissances }\end{array}$ & Pourcentage \\
\hline 1 & 39 & 560 & 7,8 \\
\hline 2 & 61 & 654 & 9,3 \\
\hline 3 & 54 & 643 & 8,3 \\
\hline 4 & 37 & 255 & 14,5 \\
\hline
\end{tabular}


Sexe

De la naissance au sevrage, 46,5 p. 100 du total des morts (191 animaux) sont des mâles et 53,5 p. 100 des femelles. Mais cette différence n'est pas significative contrairement à ce que l'on constate sur l'ensemble de la mortalité.

\section{Mortalité entre 6 mois et 2 ans}

\section{Influence $d u$ poids au sevrage}

Dans les conditions alimentaires précaires de la zone sylvo-pastorale, la période après le sevrage est difficile. Seuls, les animaux ayant acquis au sevrage un poids suffisant auront les meilleures chances de survivre. Ainsi, en comparant la moyenne des poids au sevrage des animaux morts entre 6 et 24 mois à celle des animaux vivants ou morts après 24 mois, il apparaît une différence significative entre ces deux groupes d'animaux $(15,6 \mathrm{~kg})$.

Cette observation rentre bien dans la ligne de la sélection appliquée au C.R.Z., puisqu'on $\mathrm{y}$ recherche les femelles qui présentent les meilleures qualités laitières, c'est-à-dire celles qui assurent à leurs produits la meilleure croissance entre la naissance et le sevrage.

\section{Epoque de la naissance}

A la lecture du tableau $\mathrm{n}^{\circ}$ VII, il apparaît que le sevrage éprouve particulièrement les animaux lorsqu'il survient au premier trimes- tre, c'est-à-dire ceux nés au $3^{e}$ trimestre. Or, c'est précisément à ce moment qu'a lieu le maximum des naissances lors d'une monte naturelle. Deux solutions sont à proposer : la première consiste à assurer durant la saison sèche une supplémentation aux animaux récemment sevrés, et la seconde à diminuer l'âge du sevrage de manière à ce que la transition entre l'alimentation lactée et l'alimentation à l'herbe se fasse pendant une période encore favorable sur le plan de la valeur des pâturages.

\section{CONCLUSIONS}

Les deux premières années de la vie des animaux sont particulièrement critiques car, 80 p. 100 des morts ont lieu pendant cette période; mais le taux de mortalité reste faible, 5 p. 100. Par ailleurs, et d'une manière générale, elle est plus faible durant les $1^{\text {er }}$ et $4^{\text {e }}$ trimestres; la fin de saison sèche et la saison des pluies se révèlent assez néfastes pour les animaux.

Cette étude permet de connaître les périodes de moindre résistance des animaux et, par conséquent, de conduire son élevage pour utiliser au mieux les conditions naturelles et de savoir à quel moment intervenir pour appliquer des mesures conservatoires, en particulier sur le plan de la supplémentation alimentaire. De plus, les différentes remarques peuvent trouver une application, en particulier dans le choix de la saison de monte.

\section{SUMMARY}

Study of cattle mortality in zootechnical Research Center of Dara (Senegal)

The annual mean rate of cattle death in C.R.Z. Dara is low: 6,21 p. $100 ; 1,9$ p. 100 dy before 6 months old, 3,25 before one year and 5 p. 100 before two years.

26 p. 100 of the deaths are less than 6 months old, 29 p. 100 between 6 and 12 months, and 80 p. 100 before two years. Birth-time, birth weight and weaning time have an influence on the death, like the season: between April and August and specially in July the death is the highest; but during this period, the births are the most numerous. Males are less sensible than females. 


\section{RESUMEN}

Estudio de la mortalidad de los bovinos en el Centro de investigaciones zootécnicas de Dara (Senegal)

El termino medio anual de mortalidad de los bovinos en el Centro de investigaciones zootecnicas de Dara es poco elevado: 6,21 p. 100 ; 1,9 p. 100 de los animales mueren antes de tener 6 meses de edad; 3,25 p. 100 antes de 1 año y 5 p. 100 antes de 2 años. 26 p. 100 de los muertos tienen menos de 50 dias, 29 p. 100 entre 6 y 12 meses, 80 p. 100 menos de 2 años de edad.

El peso, la epoca de nacimiento y el peso al destete tienen una influencia cierta sobre dicha mortalidad, asi como la estación : entre april y agosto y sobretodo en julio es cuando la mortalidad es más elevada; pero este periodo corresponde a el cuando se encuentra el maximo de nacimientos. Por otra parte, los machos son menos sensibles.

Las principales causas de mortalidad son los accidentes septicémicos ocurriendo después del destete.

\section{BIBLIOGRAPHIE}

L. DENIS (J. P.) et VALENZA (J.). Le comportement pondéral des femelles adultes de race Gobra. Comparaison avec les animaux importés Pakistanais et Guzera. Rev. Elev. Méd. vét. Pays trop., 1970, 23 (2) : 229-241.
2. Rapports annuels du Centre de Recherches zootechniques de Dara-Djoloff (Sénégal), 1954 à 1969. 